(C) 2017 IEEE. Personal use of this material is permitted. Permission from IEEE must be obtained for all other uses, in any current or future media, including reprinting/republishing this material for advertising or promotional purposes, creating new collective works, for resale or redistribution to servers or lists, or reuse of any copyrighted component of this work in other works. 


\title{
Compact, Omni-Directional, Circularly-Polarized mm-Wave Antenna for Device-to-Device (D2D) Communications in Future 5G Cellular Systems
}

\author{
Wei Lin and Richard W. Ziolkowski \\ Global Big Data Technologies Centre, \\ University of Technology Sydney, \\ Ultimo 2007, Sydney, Australia, \\ Email: Wei.Lin@uts.edu.au, Richard.Ziolkowski@uts.edu.au
}

\begin{abstract}
A simple, compact, omni-directional, circularlypolarized (CP) millimeter-wave antenna for Device-to-Device (D2D) communications in the next generation (5G) cellular systems is reported. It is a CP omni-directional antenna operating at $28 \mathrm{GHz}$ for mobile terminals. The antenna combines a vertical electric monopole element with four magnetic elements to coherently excite parallel electric and magnetic dipoles. This combination generates the omni-directional $C P$ radiation. The overlapping -10-dB impedance and $3-\mathrm{dB}$ axial ratio (AR) bandwidth is from 27 to $28.5 \mathrm{GHz}$, which covers the $28 \mathrm{GHz}$ frequency band proposed for $5 \mathrm{G}$ mobile cellular networks (i.e., from 27.5 to $28.35 \mathrm{GHz}$ ). The antenna has an omni-directional radiation pattern at $28 \mathrm{GHz}$ whose peak realized RHCP gain is $2.08 \mathrm{dBic}$ and whose 3-dB AR beamwidth is wide, from elevation angles $3^{\circ}$ to $136^{\circ}$. Mass production of the antenna is possible by PCB manufacturing technologies. The overall size is $3.44 \mathrm{~mm} \times$ $3.44 \mathrm{~mm} \times 1 \mathrm{~mm}(\mathrm{ka}=1.1017)$. Consequently, it could be embedded in many current popular, smart wireless devices such as cell phones, laptops, digital watches, and smart glasses as well as their future versions for operation in $5 \mathrm{G}$ cellular networks.
\end{abstract}

Index Terms - 5G cellular networks, Device-to-Device (D2D) communications, millimeter-wave antennas, omni-directional patterns, circular polarization

\section{Introduction}

Device-to-Device (D2D) communication is an evolutionary technology anticipated for future $5 \mathrm{G}$ cellular networks. It will bring significant improvements in system capacity, spectral efficiency, communication range and channel reliability as a consequence of its advantages for realizing spatial diversity [1] Figure 1 describes two types of D2D communications proposed for 5G system: data replay and direct D2D communications. In these D2D applications, portable wireless devices must be capable of communicating with any other nearby devices. Within this scenario, CP antennas with omnidirectional patterns are key components. They will provide superior radiation coverage and will avoid polarization

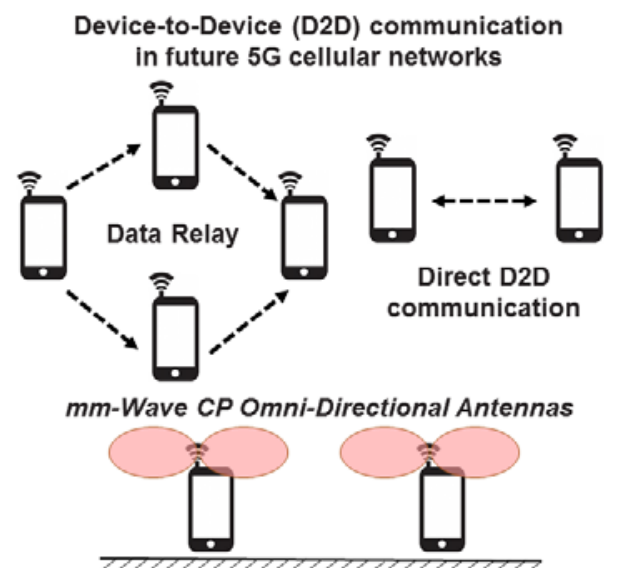

Fig. 1. Application diagram of mm-Wave omni-directional CP antennas for D2D communications in $5 \mathrm{G}$ cellular networks.

mismatches which occur in devices with only LP performance [2] - [4].

It is well-known that $5 \mathrm{G}$ broadband cellular networks have already been planned which will utilize the millimeter wave (mm-Wave) frequency spectrum. Experimental results have proved that the $28 \mathrm{GHz}$ frequency band is suitable for such 5G mm-Wave cellular systems [5]. Thus, compact omnidirectional $\mathrm{CP}$ antennas operating at $28 \mathrm{GHz}$ are very good candidates for D2D applications in 5G cellular networks.

Although some CP omni-directional antenna designs can be found at GPS or WLAN bands [6], [7], they cannot be simply rescaled to mm-Wave bands due to physical constraints. To date, only one reported design [8] realizes CP omnidirectional radiation at $37 \mathrm{GHz}$ in the mm-Wave band. However, its size is quite large (diameter: $38 \mathrm{~mm}=4.7 \lambda_{0}$ ). Consequently, it is not suitable to embed it into small portable devices like smart watches and glasses.

As the first of its kind, we will demonstrate a simple, compact (diameter: $3.14 \mathrm{~mm}=0.32 \lambda_{0}$ ) mm-Wave omnidirectional CP antenna at $28 \mathrm{GHz}$. Its performance characteristics confirm that is an outstanding candidate for D2D applications in future 5G cellular networks. 


\section{Antenna Design and Performance}

\section{A. Antenna Design and Operating Principle}

The reported antenna is shown in Fig. 2. It is printed on a single piece of Rogers Duroid ${ }^{\mathrm{TM}} 5880$ substrate whose thickness is $1 \mathrm{~mm}$ and whose relative dielectric constant is 2.2. The center conductor of the $50 \Omega$ coaxial feed line (Type 04750 from Shenzhen Kansai Industrial LTD) acts as a vertical monopole. Four meandered arms are connected to it at its apex. Each arm has pieces traversing the top and bottom surfaces of the substrate that are connected by a plated via. An annular metallization layer is enclosed by the bottom strips of the four arms and is connected directly to the outer surface of the coax. The current distributions on all five elements are shown in Figure 3. The loop currents form a vertical magnetic dipole, which is parallel to the electric dipole formed by the monopole, and in phase with it. As a result, omni-directional CP radiation is generated.

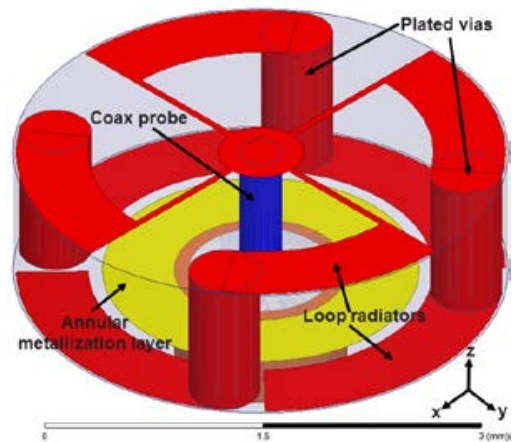

Fig. 2. Antenna configuration.

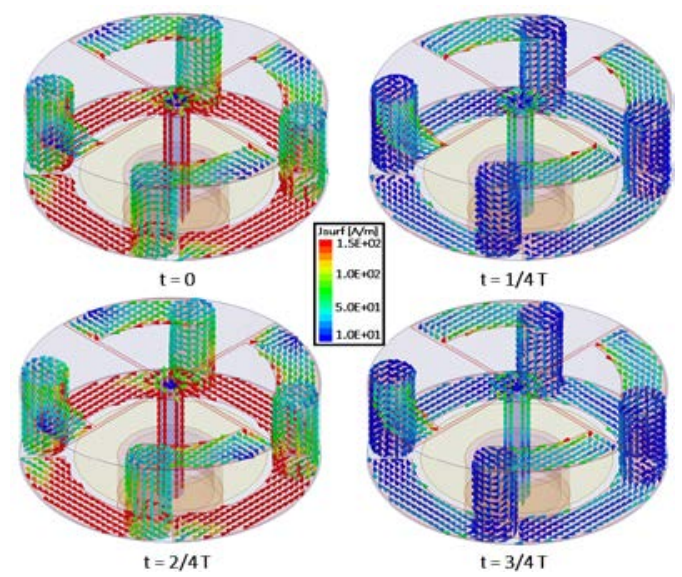

Fig. 3. Current distributions within one source period at $28 \mathrm{GHz}$.

\section{B. Antenna Performance}

The antenna exhibits very good radiation performance. As shown in Fig. 4, the overlapping -10-dB impedance and 3-dB AR bandwidth is from 27 to $28.5 \mathrm{GHz}$, which covers the 28 $\mathrm{GHz}$ frequency band, 27.5 to $28.35 \mathrm{GHz}$, proposed for $5 \mathrm{G}$ systems. Its realized gain patterns at $28 \mathrm{GHz}$ are given in Fig. 5 . Very good omni-directional CP radiation patterns are observed. The peak realized RHCP gain is 2.08 dBic. The $3-\mathrm{dB}$ AR beamwidth in the $\phi=0^{\circ}$ azimuth plane is also wide, from the elevation angles $3^{\circ}$ to $136^{\circ}$.

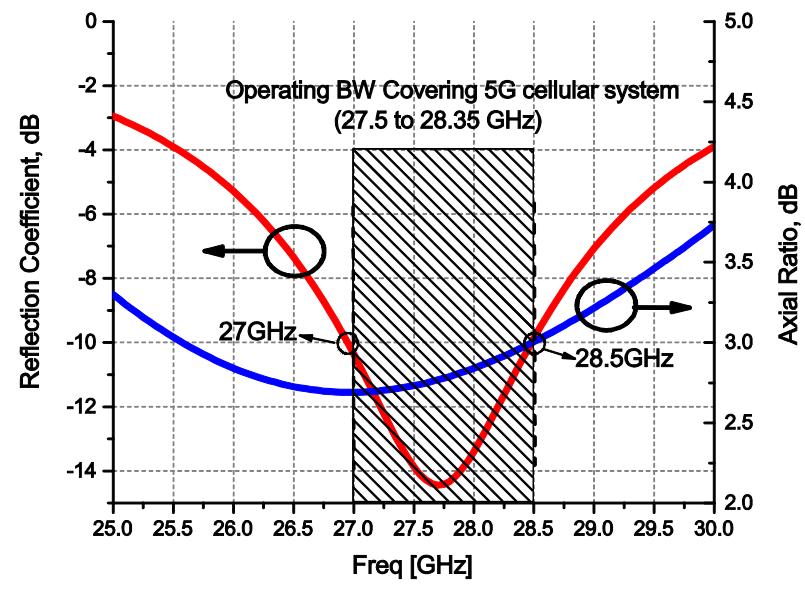

Fig. 4. Simulated reflection coefficient and AR bandwidth.

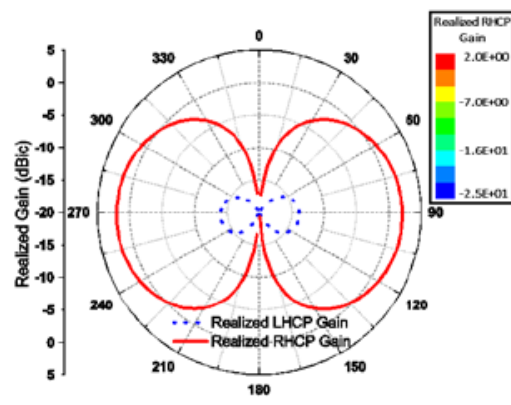

(a)

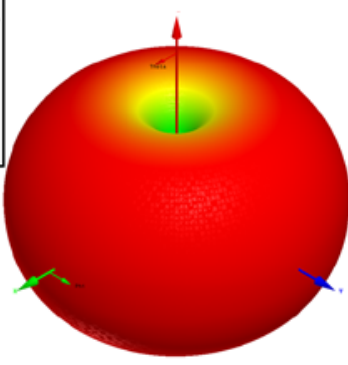

(b)
Fig. 5. Simulated realized gain patterns at $28 \mathrm{GHz}$. (a). RHCP and LHCP 2D patterns in the $\phi=0^{\circ}$ plane; and (b) RHCP 3D pattern.

\section{References}

[1] M. N. Tehrani, M. Uysal and H. Yanikomeroglu, “Device-to-Device communication in 5G cellular networks: challenges, solutions, and future directions,” IEEE Commun. Mag., vol. 52, no. 5, Feb. 2014, pp. 86-92.

[2] W. Lin and H. Wong, "Multi-polarization reconfigurable circular patch antenna with L-shaped probes,” IEEE Antennas Wireless Propag. Lett., publication in process, 2017.

[3] H. Wong, W. Lin, L. Huitema and E. Arnaud, "Multi-polarization reconfigurable antenna for wireless biomedical system," IEEE Trans. Biomed. Circuits Syst., publication in process, 2017.

[4] W. Lin and H. Wong, "Polarization reconfigurable multi-slot antenna for body-centric wireless communication system," IEEE International Workshop on Electromagnetics (IWEM), May, 2016

[5] T. S. Rappaport et al., "Millimeter wave mobile communications for 5G cellular: It will work!” IEEE Access, vol. 1, pp. 335-349, 2013.

[6] B. Li and Q. Xue, "Omnidirectional circularly polarized antenna combining monopole and loop radiators,” IEEE Antennas Wireless Propag. Lett., vol. 12, pp. 607-610, 2013.

[7] Y. F. Yu, Z. X. Shen and S. L. He, "Compact omnidirectional antenna of circular polarization,” IEEE Antennas Wireless Propag. Lett., vol. 11, pp. 1466-1469, 2012.

[8] J. M. Fernandez, J. L. Masa-Campos, and M. Sierra-Perez, "Circularly polarized omnidirectional millimeter wave monopole with parasitic strip elements,” Microw. Opt. Technol. Lett., vol. 49, pp. 664 668, Mar., 2007. 